\title{
Manejo perioperatorio de tumores intracraneales: rol del neurocirujano
}

\author{
Perioperative management of intracranial tumours: \\ the neurosurgeon's role \\ C. Polo-Torres, H.R. Alvis-Miranda, R. Villa-Delgado, L.R. Moscote-Salazar
}

\section{RESUMEN}

El manejo perioperatorio de los pacientes con tumores cerebrales es un reto para el neurocirujano y todo el equipo quirúrgico. El médico debe considerar factores como el tipo de tumor, la extensión de la enfermedad, el tratamiento recibido, la presencia de comorbilidades y el pronóstico de la patología. La ejecución correcta de todos los aspectos implicados en el manejo perioperatorio en pacientes con tumores intracraneales contribuirá a prolongar la vida y a mejorar la calidad de vida de los pacientes.

Palabras clave. Tumores cerebrales. Evaluación preoperatoria. Neurocirugía.

\begin{abstract}
The perioperative management of patients with brain tumours is a challenge for the neurosurgeon and the entire surgical team. The treating physician should consider factors such as the type of tumour, extent of disease, treatment received, the presence of comorbidities and prognosis of the disease itself. The successful execution of all aspects involved in perioperative management in patients with brain tumours will help prolong the life and improve the quality of life of patients.
\end{abstract}

Key words. Brain tumors. Preoperative evaluation Neurosurgery.
Universidad de Cartagena. Cartagena de Indias. Colombia.

Recepción: 30 de diciembre de 2012

Aceptación provisional: 22 de febrero de 2013

Aceptación definitiva: 18 de marzo de 2013

\section{Correspondencia:}

Luis Rafael Moscote-Salazar

Universidad de Cartagena

Cartagena de Indias

Colombia

E-mail: mineurocirujano@aol.com 


\section{INTRODUCCIÓN}

La primera descripción exitosa de cirugía para extirpar un tumor cerebral fue realizada por el vanguardista cirujano francés Louis en $17741^{1}$. Gracias a los avances realizados por Harvey Cushing en cirugía para el tratamiento de tumores cerebrales a inicios de 1900, se abrió una etapa en la neurocirugía oncológica ${ }^{2}$, convirtiéndose en una gran parte de la neurocirugía.

Los tumores del sistema nervioso central (SNC) representan un grupo de entidades diversas en morfología, ubicación, biología molecular y comportamiento clínico $^{3}$. En Europa, la incidencia estandarizada (mundial) de tumores primarios del SNC varía entre 4,5 y 11,2 casos por cada 100000 hombres y de 1,6 a 8,5 casos por cada 100.000 mujeres ${ }^{4}$. En Sudamérica, la incidencia es de 2,4 por cada 100.000 hombres y de 1,9 por cada 100.000 mujeres $^{4}$. La incidencia de tumores intracraneales se ha incrementado con respecto al comportamiento epidemiológico, en parte debido al descubrimiento de nuevas tecnologías de imagen como la tomografía computarizada (TAC) y la resonancia magnética (RM) ${ }^{2,5}$.

La mayoría de los tumores son gliomas, con una tasa de incidencia ajustada para la edad entre $-0,5 \%$ a $-0,8 \%(\mathrm{p} \leq 0,034)$; se ha observado un incremento particular de éstos en el lóbulo frontal con una tasa de incidencia ajustada para la edad de $+1,4 \%$ $\mathrm{a}+1,7 \%(\mathrm{P} \leq 0,012)^{6}$. Los tumores de origen astrocítico, oligodendrocítico y ependimal representan entre el 70 y el $75 \%$ de estos tumores $^{7,8}$. Como hallazgo fortuito en la evaluación de RM por otra causa, los tumores cerebrales benignos representan un 1,6\%, siendo los meningiomas los más frecuentemente encontrados 9 .

Cada tumor cerebral consta de su propia biología, pronóstico y tratamiento, aunque cada tumor se desarrolla a partir del crecimiento anormal de un tipo de célula específico y pese a que otros no se deriven del tejido cerebral (por ejemplo, los meningiomas y linfomas), para la mayoría de los tumores intracraneales, la presentación clínica, el abordaje diagnóstico, y el tratamiento inicial son similares ${ }^{10}$. Se usan diversos tratamientos ${ }^{11,12}$, que dependen del tamaño, ubicación, tipo de tumor, velocidad de crecimiento y el estado general del paciente. Las opciones de tratamiento incluyen cirugía, radioterapia y quimioterapia, agentes biológicos dirigidos o una combinación de éstas $^{13-18}$. Sin embargo, la resección quirúrgica (después de haber evaluado el riesgo del tumor), es por lo general la primera recomendación de tratamiento para reducir la presión intracraneal rápidamente ${ }^{19-29}$.

Los problemas más frecuentes que se presentan en los tumores cerebrales son las convulsiones, el edema peritumoral, los efectos secundarios de la medicación usa$\mathrm{da}$, y el tromboembolismo venoso ${ }^{30}$. Teniendo en cuenta que la intervención quirúrgica es por lo general una de las conductas iniciales, es necesario tener presente cada uno de los problemas mencionados. Este artículo revisa brevemente el manejo perioperatorio de los tumores intracraneales y las posibles complicaciones en el postoperatorio (POP), involucrando la preparación general, el tratamiento de convulsiones, de la hipertensión endocraneal y del edema peritumoral, del tromboembolismo venoso y algunos aspectos metabólicos del paciente con tumor intracraneal.

\section{EVALUACIÓN PREOPERATORIA}

La evaluación preoperatoria es una función esencial del servicio de Anestesiología y es un error limitarla sólo a una evaluación rápida y somera del paciente y al pedido de exámenes complementarios. Todo paciente que va a ser sometido a una intervención debe tener su evaluación preanestésica, siendo sus objetivos la confección de una historia clínica completa, un examen físico minucioso, y valorar los exámenes complementarios. Además, en esta consulta, el anestesiólogo debe hacerse familiar con todos los estudios realizados al paciente. Es muy importante la monitorización neurológica intraoperatoria, de acuerdo con el neurocirujano ${ }^{31}$.

La mayoría de los tumores intracraneales se descubren cuando aparecen signos y síntomas de efecto de masa, el paciente convulsiona o de forma accidental. Es común que los pacientes presenten cefalea, 
clínica similar a la enfermedad cerebrovascular isquémica, vómitos, alteraciones visuales, alteración de la función cognitiva, ataxia, entre otras. Si la presión intracraneal (PIC) se eleva, aparecerá hipertensión, bradicardia, arritmias cardiacas, anormalidades electrocardiográficas y se incrementa el riesgo de que el paciente desarrolle síndromes de herniación cerebral.

Todo el cuadro clínico junto con los déficits neurológicos debe establecerse en la historia clínica con el fin de poder hacer comparaciones en los distintos momentos del tratamiento del paciente y en el POP. Hay que establecer todo tratamiento previo recibido para el manejo de la patología de base, puesto que los agentes quimioterapéuticos pueden afectar la función de ciertos órganos.

La dexorrubicina y la daunorubicina pueden generar arritmias cardiacas e insuficiencia cardiaca (IC) congestiva ${ }^{32-35}$. La bleomicina y el busulfan pueden generar fibrosis pulmonar significativa, especialmente en presencia de altas concentraciones de oxígeno ${ }^{36-40}$. El carboplatino y el cisplatino pueden generar disfunción e incluso insuficiencia renal ${ }^{41-44}$, la ifosfamida y la ciclofosfamida pueden conllevar a cistitis hemorrágica ${ }^{45-48}$, además, la ciclofosfamida puede inhibir significativamente la acción de la acetilcolinesterasa plasmática ${ }^{49-53}$. La mayoría de los agentes quimioterapéuticos generan disfunción hepática transitoria y pueden alterar la farmacocinética de diversos agentes anestésicos ${ }^{54-56}$. En función de los hallazgos, estarán indicadas las distintas pruebas que evaluarán tales alteraciones. Es de destacar que se debe continuar la medicación anticonvulsiva y esteroidea.

En cuanto a las experiencias quirúrgicas previas se debe interrogar sobre dificultades y complicaciones ${ }^{30}$.

Con base en esto y según la actualización de la evaluación cardiovascular perioperatoria para cirugía no cardíaca de la Sociedad de Anestesiología Cardiovascu$\operatorname{lar}^{57-58}$, se indica que la historia inicial, la evaluación del examen físico y el electrocardiograma (ECG) deben centrarse en la identificación de trastornos cardíacos potencialmente graves, como enfermedad co- ronaria (por ejemplo, infarto de miocardio previo (IM) y angina de pecho), IC, arritmias sintomáticas, la presencia de marcapasos o un desfibrilador implantable, o una historia de intolerancia ortostática. También es importante identificar la presencia de anemia porque ésta puede aumentar el riesgo de complicaciones en el $\mathrm{POP}^{30-59}$. Algunos aspectos a tener en cuenta:

- Hábito corporal: grado de obesidad, alteraciones musculoesqueléticas. En el caso del paciente con lesiones metastásicas en cerebro, el examen físico revelará alteraciones constitucionales como caquexia.

- Aparato respiratorio: apertura bucal, obstrucciones, infecciones agudas o crónicas. Detectar posibles problemas de intubación, estado de las piezas dentarias, semiología facial. Es posible encontrar sibilancias, o sonidos pulmonares disminuidos a la auscultación, o piel pigmentada ${ }^{60}$.

- Sistema cardiovascular: es indispensable la evaluación de los signos vitales (incluyendo tensión arterial en ambos brazos), pulso carotídeo, soplos cardíacos, presión venosa y pulsaciones yugulares, palpación precordial, arritmias, cianosis o disnea, evaluación de las extremidades en búsqueda de edema e integridad vascular.

- Examen neurológico: cualquier anormalidad neurológica es posible debido a que los tumores pueden ubicarse en cualquier lugar del cerebro. Como la mayoría se inicia focalmente causarán cambios locales, por lo que la debilidad muscular, el entumecimiento, la descoordinación o la hiperreflexia en extremidades junto con los cambios visuales, serán puntos clave ${ }^{60}$.

Es importante la evaluación del estado funcional del paciente según la escala Karnofsky (KPS; Tabla 1) ${ }^{59-61}$. Referente a esto, existe el estudio realizado por Ianssen y $\mathrm{col}^{62}$ que tuvo como finalidad analizar el pronóstico de la cirugía y la terapia coadyuvante en pacientes con tumores cerebrales malignos, en el cual incluyeron un total de 186 pacientes, tratados en una sola institución en un período de 6,5 años, donde 172 fue- 
ron operados. Los factores más importantes relacionados con la supervivencia fueron las reintervenciones quirúrgicas, la KPS, la extensión de la resección y la edad del paciente. Los pacientes con glioblastoma multiforme mostraron una supervivencia prolongada de 10 meses después de resecciones repetidas. Con base en el KPS, $68 \%$ de los pacientes eran independientes antes de la operación, el 13\% parcialmente dependiente y el $9 \%$ dependiente. Después de la operación, el $81 \%$ eran independientes, $12 \%$ parcialmente dependiente y el $7 \%$ dependiente. Los pacientes con KPS 80-100 habían mejorado la supervivencia en comparación con KPS <80. Los pacientes dependientes tuvieron un mejor resultado (KPS antesdespués) en comparación con los pacientes con KPS mayor $(p<0,0001)$. Sin embargo, los tumores de localización central se correlacionan con un mal resultado neurológico
( $p<0,01$ ). Estos pacientes obtuvieron una puntuación media de 16 puntos menos en la KPS después de la operación en comparación con antes. Con base en los resultados en este estudio se alude que el número de casos es muy pequeño para emitir conclusiones; sin embargo, sugieren que la cirugía se debería realizar de la forma más radical posible, y la resección repetida se debería considerar solo si se confirma recurrencia. La KPS al ingreso fue un buen predictor de la supervivencia, según lo recogido y se comprobó que un KPS $\geq 80$ es un factor de buen pronóstico; se encontraron diferencias significativas en la supervivencia de los pacientes con KPS de 90 o 80 en todos los pacientes. Con esto podemos inferir que hoy por hoy la KPS puede seguir siendo de gran ayuda para la evaluación neurológica y de la calidad de vida del paciente antes y después de una cirugía intracraneal ${ }^{62}$.

Tabla 1. Escala de estado funcional de Karnofsky ${ }^{61,63}$

\begin{tabular}{ll}
\hline $100 \%$ & Normal, sin quejas; sin evidencia de enfermedad \\
\hline $90 \%$ & Actividades normales; signos y síntomas leves de enfermedad \\
\hline $80 \%$ & Actividad normal con esfuerzo; algunos signos y síntomas de enfermedad \\
\hline $70 \%$ & Capaz de cuidarse; incapaz de llevar a término actividades normales o trabajo activo \\
\hline $60 \%$ & Requiere atención ocasional, pero puede atender la mayoría de sus necesidades personales \\
\hline $50 \%$ & Requiere gran atención y cuidado médico frecuente. Encamado menos del 50\% del día \\
\hline $40 \%$ & Discapacitado; necesita cuidados y atenciones especiales. Encamado más del $50 \%$ del día \\
\hline $30 \%$ & Severamente discapacitado, está indicada la hospitalización, aunque la muerte no es inminente \\
\hline $20 \%$ & Paciente muy grave; necesita hospitalización y tratamiento de soporte \\
\hline $10 \%$ & Moribundo; el proceso fatal progresa rápidamente \\
\hline $0 \%$ & Muerte \\
\hline
\end{tabular}

Realizar el plan quirúrgico para abordar un tumor cerebral puede resultar complicado. A menudo, la variable definitoria es la ubicación del tumor. Gracias a la tecnología, el tratamiento quirúrgico de los tumores intracraneales ha avanzado. Así, tumores intraaxiales de ubicación profunda, que necesitan abordajes transcorticales deberían realizarse con neuronavegación estereotáxica intraoperatoria con imagen funcional (fMRI), imagen con tensores de difusión, tractografías, y algunas veces espectroscopía de resonancia magnética (MRS). La tractografía puede ayudar a evitar daño a estructuras axonales como las radiaciones ópticas, el fascículo arqueado, mientras que la MRS puede ayudar en la elección del sitio para biopsiar ${ }^{64-75}$, sin embargo, todas estas medidas no están universalmente disponibles y son complejas en su procesamiento.

En la consulta preoperatoria deberán establecerse todos los objetivos de la cirugía: el establecer o confirmar un diagnós- 
tico, descomprimir estructuras nerviosas, estabilizar, reconstruir o curar. Es importante la discusión prequirúrgica de estos objetivos con el paciente y los familiares para evitar falsas expectativas.

\section{MANEJO PERIOPERATORIO DE EDEMA CEREBRAL Y DE LA HIPERTENSIÓN INTRACRANEANA}

El edema peritumoral es una seria complicación del manejo de las neoplasias intracraneales ${ }^{76}$ que, en función de la severidad y el grado, puede dificultar los procedimientos quirúrgicos. Va asociado generalmente a tumores de comportamiento maligno ${ }^{77}$, ya que la vasculatura tumoral carece de las uniones fuertes ("tight junctions") que se hallan en la microvasculatura cerebral, es decir, tienen una barrera hematoencefálica incompleta. Los tumores metastásicos y algunos tumores primarios como el glioblastoma multiforme producen cerca de $90 \mathrm{ml}$ de fluido por día. Tal exceso debe acomodarse junto a los demás volúmenes intracraneales, es decir, a los 1.100$1.300 \mathrm{ml}$ del volumen del espacio intracelular y a los 100-150ml del intersticial, a los 75-100 ml del LCR y a una cantidad similar de sangre $^{78}$. El cerebro produce cerca de $30 \mathrm{ml}$ de agua. Atendiendo a la doctrina de Monroe-Kellie, incrementos adicionales a los volúmenes básicos intracraneales producirá un desplazamiento inicial del fluido desde los compartimientos del LCR $(\approx 10$ $\mathrm{mmHg})$ y venoso $(\approx 10 \mathrm{mmHg})$, ambos de baja presión, hacia el compartimiento arterial, de alta presión $(\approx 100 \mathrm{mmHg})^{78}$.

En condiciones normales, un efecto "sumidero" es proporcionado por los ventrículos y el líquido cefalorraquídeo subaracnoideo para permitir la circulación constante y la renovación del espacio extracelular. Este efecto "sumidero" provoca acumulación de líquido extracelular debido a que la principal fuerza conductora del edema vasogénico hacia el espacio extracelular es el gradiente de presión hidrostática (presión arterial sistémica - PIC ${ }^{78}$.

La conducta más antigua es el uso de corticoides; sin embargo, su efecto aún no está bien aclarado, pero se infiere que ellos pueden reducir la permeabilidad capilar del tumor. Usualmente los pacientes con edema peritumoral requieren la administración prequirúrgica de dexametasona, a la dosis mínima efectiva, a dosis carga de $10 \mathrm{mg}$, seguido de $16 \mathrm{mg} /$ día en varias do$\mathrm{sis}^{79}$, la dosis puede aumentarse hasta 100 $\mathrm{mg} /$ día si es necesario. Ayudan a disminuir los síntomas ya que reducen el edema vasogénico y el efecto de masa. Además, ayudan con el manejo del edema producto del trauma quirúrgico, facilitando la cirugía. Deben prescribirse junto con un inhibidor de bomba de protones para proteger la mucosa gastrointestinal y evitar la aparición de úlceras por estrés.

El edema no solo causa un efecto de masa, sino un aumento de la presión intracraneal, lo cual conduce a trastornos neurológicos mediante la interrupción de la homeostasis del tejido y la reducción del flujo sanguíneo local. Para el manejo de la HIC, puede requerirse manitol si se presenta incremento de la presión intracraneana que causa deterioro del estado de conciencia. El manitol actúa como diurético osmótico, ayudando a reducir el edema cerebral atrayendo agua hacia el espacio intravascular. Debe usarse solo en caso de absoluta emergencia, puede aportar un período de ventana que permitiría llevar al paciente a la unidad neuroquirúrgica ${ }^{79}$.

\section{MANEJO DE LAS CONVULSIONES}

No hay aspecto más controvertido dentro del manejo de los tumores intracraneales que lo relacionado con la profilaxis anticonvulsiva. Cerca de un $30-50 \%$ de los pacientes con tumores cerebrales debutan con convulsiones como síntoma inicial ${ }^{80,81}$. El riesgo de convulsionar varía de acuerdo al tipo de tumor, su grado y ubicación ${ }^{82,83}$. Diversos estudios retrospectivos no han podido demostrar beneficios de la profilaxis anticonvulsivante ${ }^{84-87} \mathrm{y}$, peor aún, se conoce que los anticonvulsivantes tienen interacciones con los corticoides y los quimioterápicos ${ }^{82,88}$. Estas convulsiones pueden ser difíciles de controlar, y pueden necesitarse varios medicamentos ${ }^{89}$. 
Las convulsiones aparecen principalmente en relación con tumores de bajo grado tipo astrocitomas y oligodendrogliomas (60-80\%) en comparación con tumores de alto grado tipo glioblastoma $(30-50 \%)^{80}$. Hay más riesgo de generar convulsiones cuanto más cercano esté a la corteza respecto a los de ubicación profunda; lo mismo que los tumores de ubicación frontal, temporal y parietal generan más convulsiones que los de ubicación occipital ${ }^{90}$, es importante reconocer que el tumor en sí no es epileptogénico, pues son lesiones eléctricamente inertes en el electroencefalograma; las anormalidades derivan de la corteza adyacente a la lesión ${ }^{91}$.

Las convulsiones son una complicación potencialmente devastadora de las resecciones tumorales, por ello muchos neurocirujanos usan profilaxis anticonvulsivante en el perioperatorio, sin embargo lo que se conoce acerca de los beneficios de esta profilaxis es poco claro y controvertido. Se ha demostrado que la radiación y la quimioterapia tienen un efecto positivo en el control de las convulsiones relacionadas con tumores ${ }^{92}$.

Con el objetivo de estimar la efectividad de la profilaxis anticonvulsivante y la tasa de efectos adversos en pacientes con tumores cerebrales, Tremont-Lukats y $\mathrm{col}^{93}$ realizaron en 2008 un metanálisis, el cual no encontró diferencias entre el grupo intervenido (fenitoína, fenobarbital y divalproato sódico) y el grupo control en lo que respecta a prevención de la primera convulsión, y el riesgo de evento adverso fue mayor en el grupo con medicación anticonvulsivante (número necesario para dañar= 3; RR 6,10, 95\% CI 1,10 a 34,63; $\mathrm{p}=0,046$ ). Los autores concluyen que la evidencia es neutra, aplicando la fenitoína, fenobarbital y divalproato sódico.

Kerrigan y col $^{94}$ en 2011 realizaron un metanálisis con el objetivo de evaluar la efectividad y la tolerabilidad de medicamentos anticonvulsivantes de común uso en adultos con tumores cerebrales. Solo encontraron un ensayo con todos los criterios de inclusión, en el cual se evaluaba la seguridad y viabilidad de cambiar fenitoína por levetiracetam o continuar fenitoína para el control de convulsiones asociadas a gliomas posterior a craneotomía ${ }^{95}$. Los autores encontraron que el levetiracetam (anticonvulsivante no inductor enzimático) era tan bien tolerado y efectivo como la fenitoína (anticonvulsivante inductor enzimático) en el tratamiento de convulsiones en pacientes con tumores cerebrales. El $87 \%$ de los pacientes con levetiracetam estaban libres de convulsiones a los 6 meses en comparación con el $75 \%$ obtenido con fenitoína.

Wu y col ${ }^{961}$ investigaron de manera prospectiva y aleatorizada el uso de fenitoína durante 7 días en la profilaxis anticonvulsivante en pacientes que son sometidos a resección supratentorial de metástasis cerebrales o gliomas versus el grupo control. Los autores encontraron una incidencia de convulsiones baja (8\% [95\% IC 3\%-18\%]) aun sin profilaxis, siendo del $3 \%$ la incidencia de convulsiones graves; además encontraron que la administración rutinaria de fenitoína se asociaba a morbilidad significativa relacionada con el medicamento. Aunque el poder del estudio es limitado y la incidencia de convulsiones baja, se cuestiona el uso rutinario de fenitoína como profilaxis en esta población de pacientes.

Estudios que evalúan el rol de la profilaxis anticonvulsivante en tipos específicos de tumores, como los meningiomas ${ }^{97}$, no han encontrado beneficio alguno en la prevención de convulsiones postquirúrgicas tempranas o tardías.

En el caso de pacientes pediátricos con tumores intracraneales, Hardesty y collo evaluaron los factores de riesgo en niños previamente libres de convulsiones para desarrollarlas en el postoperatorio; encontraron que estaban asociadas con tumores de ubicación supratentorial, edad $<2$ años, e hiponatremia postquirúrgica; no hubo relación con la patología y tamaño tumoral, ni con estar ubicado en el área frontotemporal. Los autores, debido a la baja incidencia de convulsiones en su serie de pacientes $>2$ años con sodio sérico normal, no recomiendan el uso rutinario de medicación anticonvulsivante profiláctica, pero en el paciente $<2$ años se debe evaluar con mayor cuidado tal necesidad. Desafortu- 
nadamente hay carencia de evidencia robusta que apoye la elección de medicación anticonvulsivante profiláctica en este tipo de pacientes. Aunque hay una tendencia a usar agentes anticonvulsivantes que no sean inductores enzimáticos, tales como el ácido valproico o valproato de sodio, lamotigrina, etosuximida, gabapentina, pregabalina, levetiracetam, tiagabine, rufinamida, lacosamida, zonisamida, vigabatrin, clobazam y midazolam; sin embargo la evidencia comparativa para justificar su uso clínico es limitada, por lo que la decisión de comenzar un medicamento anticonvulsivante en forma de profilaxis está en función de la evaluación de los factores de riesgo individuales y de la discusión cuidadosa con los pacientes y sus familiares ${ }^{93,94}$. Por ejemplo, se podrían prescribir en pacientes cuyo tumor está adyacente a la corteza cerebral, especialmente en las áreas frontal y temporal y preferiblemente que el anticonvulsivante no sea inductor enzimático, para evitar interacciones con otros medicamentos usados $^{11}$.

\section{MANEJO DE TROMBOEMBOLISMO VENOSO (TEV)}

Trousseau en 1865 fue el primero en describir la tendencia a desarrollar TEV los pacientes con neoplasias ${ }^{99}$. Se considera, que en general, el cáncer incrementa el riesgo de TEV unas 4.1 veces, y la quimioterapia lo hace en 6,5 veces $^{100,101}$. El TEV es una complicación frecuente y una de las principales causas de muerte en el paciente con tumor cerebral ${ }^{102,103}$. Cerca del $34 \%$ de estos pacientes desarrolla TEV en el curso de su enfermedad ${ }^{104,105}$; a pesar de ello la terapia anticoagulante se asocia con temor por el riesgo de sangrado, especialmente de hemorragia intracraneal ${ }^{106,107}$. A pesar de que la evidencia muestra que tumores, como el cerebral o el pancreático se asocian con alto riesgo de desarrollar TEV, las guías clínicas recomiendan tratamiento preventivo para VTE en pacientes con cáncer únicamente cuando están hospitalizados para recibir tratamiento médico o quirúrgico, pero no de manera ambulatoria ${ }^{108}$.
La anticoagulación es una importante contraindicación en neurocirugía. Cualquier sangrado en la cavidad tumoral puede conllevar a consecuencias desastrosas y daño neurológico permanente. El ácido acetilsalicílico y otros agentes antiagregantes plaquetarios deben suspenderse 7-10 días antes de la cirugía. El INR se debe normalizar en los que consumen warfarina. Como profilaxis antitrombótica se puede suministrar a dosis bajas heparina de bajo peso molecular.

El paciente neuroquirúrgico es considerado de alto riesgo para el TEV, pero también está en igual riesgo para desarrollar hemorragias $^{109}$. Las guías $\mathrm{ACCP}^{110}$ establecen que el paciente que va a ser sometido a un abordaje neuroquirúrgico significativo debería recibir tromboprofilaxis de manera rutinaria junto con compresión neumática intermitente (CNI). Se acepta el uso de heparina de bajo peso molecular o heparina no fraccionada a bajas dosis ${ }^{111}$. Se recomienda considerar para estos pacientes una evaluación con TAC cerebral en el día siguiente a la intervención para descartar sangrados, antes de iniciar la terapia profiláctica. Si el paciente tiene un riesgo particularmente alto para desarrollar trombosis se recomienda hacer combinación del método farmacológico con un mecánico ${ }^{111}$.

La heparina de bajo peso molecular como profilaxis inicial ha demostrado ser segura y efectiva ${ }^{112}$; en la tabla 2 se exponen las dosis y los medicamentos recomendados para cirugía de alto riesgo en pacientes con función renal normal. Desafortunadamente los estudios sobre los nuevos medicamentos antitrombóticos en relación al TEV incluyen pocos pacientes con cáncer y solo hay evaluación en el contexto clínico específico para el fondaparinaux e idraparinux ${ }^{113}$.

De interés, Khemasuwan y col $^{114}$ en su estudio tipo caso-control para evaluar retrospectivamente el impacto del uso de estatinas en relación a la ocurrencia de TEV en pacientes con tumores sólidos $(n=740)$, observaron que en el grupo de pacientes que recibió estatinas $(n=194)$ solo el $8 \%$ desarrolló TEV versus $21 \%$ en el grupo control $(\mathrm{n}=546)(\mathrm{OR} 0,33$; IC 95\%, 0,19-0,57); sugi- 
riendo que el uso de estos medicamentos se asocian con reducción significativa en la ocurrencia de TEV. Esto es de especial interés en el paciente que tiene sangrado intracraneal activo en el que la anticoagulación está contraindicada ${ }^{115}$ o en aquellos con metástasis cerebrales en los que se requiere una monitorización estricta, colocando a las estatinas como medicación alternativa en este grupo de pacientes.

Tabla 2. Dosis y medicamentos recomendados para cirugía de alto riesgo en pacientes con función renal normal. Modificado de Muntz y Michota ${ }^{111}$

\begin{tabular}{ll}
\hline \multicolumn{1}{c}{ Medicación } & \multicolumn{1}{c}{ Dosificación } \\
\hline Heparina no fraccionada a baja dosis & $5,000 \mathrm{U} \mathrm{SC} / 8 \mathrm{~h}$ \\
\hline \multirow{2}{*}{ Heparina de bajo peso molecular } & Enoxaparina $40 \mathrm{mg} /$ día $-\mathrm{SC}$ \\
& Dalteparina 5,000 IU/día - SC \\
& Tinzaparina 3,500 IU/día - SC \\
\hline Inhibidor del factor Xa & Fondaparinux 2,5 mg/día - SC \\
\hline
\end{tabular}

SC: subcutáneo

\section{MANEJO METABÓLICO}

Como ha sido mencionado, la presencia de tumores intracraneales, especialmente tumores malignos primarios de cerebro representan una enfermedad devastadora. La mayoría de estos pacientes recibe corticosteroides de forma perioperatoria, específicamente dexametasona. Este esteroide reduce el edema relacionado con el tumor y el debido al trauma quirúrgico o la radiación eleva la glicemia ${ }^{116,117}$. La hiperglicemia se relaciona con peor pronóstico ${ }^{118-121}$.

Uno de los efectos de la terapia con radiación es que genera un estado oxidativo en el tejido junto con necrosis ${ }^{122-124}$; como consecuencia de esto se libera glutamato $^{125}$. Este neurotransmisor es excitotóxico; rápidamente se metaboliza a glutamina gracias a los astrocitos ${ }^{125,126}$; la glutamina es también una fuente energética importante tanto para las células tumorales como para los macrófagos asociados a éste ${ }^{127-130}$.

Con el objetivo de contrarrestar los diferentes estragos metabólicos que genera la presencia del tumor, pero manteniendo el estado nutricional del paciente, se recomienda la dieta cetogénica, es decir, cambiar el sustrato principal para el metabolismo energético cerebral por los cuerpos cetónicos. Lo mismo sucede con la restricción dietaria, la cual reduce glicemia y ele- va la concentración de los cuerpos cetónicos, previniendo el crecimiento tumoral y por ende reduciendo la inflamación asocia$\mathrm{da}^{131-134}$.

\section{CONSIDERACIONES FINALES}

El manejo perioperatorio se desglosa en una serie de etapas críticas para el buen desarrollo del acto quirúrgico global. Desde el mismo momento en que se decide la intervención por parte del neurocirujano, de haberse considerado el tipo de tumor, la vía de abordaje, la edad del paciente, el estado neurológico y las comorbilidades, el proceso debe ser estrictamente coordinado por el neurocirujano y el equipo que realizaran la cirugía. Es importante mencionar que debe evaluarse siempre el impacto y secuelas neurológicas que potencialmente sufriría el paciente ante la realización de la cirugía. El cumplimiento de esta serie de etapas permitirá un mejor desenlace y una mayor satisfacción para el neurocirujano, el paciente y su familia.

\section{BIBLIOGRAFÍA}

1. BAKAY L. Cruveilhier on meningiomas (18291842). Surg Neurol 1989; 32: 159-164. 
2. KIm Y-H, Song SW, LeE JY, KIm JW, KIM YH, PHI $\mathrm{JH}$ et al. Surgically treated brain tumors: a retrospective case series of 10,009 cases at a single institution. World Neurosurg 2011; 76 : 555-563.

3. Wrensch M, Minn Y, Chew T, Bondy M, Berger MS. Epidemiology of primary brain tumors: current concepts and review of the literature. Neuro Oncol 2002; 4: 278-299.

4. Ferlay J, Shin HR, Bray F, Forman D, Mathers C, PARKIN DM. Estimates of worldwide burden of cancer in 2008: GLOBOCAN 2008. Int J Cancer 2010; 127: 2893-2917.

5. Arora RS, Alston RD, Eden TOB, Estlin EJ, MoRAN A, GERACI M et al. Are reported increases in incidence of primary CNS tumours real? An analysis of longitudinal trends in England, 1979-2003. Eur J Cancer 2010; 46: 1607-1616.

6. Zada G, Bond AE, Wang YP, Giannotta SL, DeaPEN D. Incidence trends in the anatomic location of primary malignant brain tumors in the United States: 1992-2006. World Neurosurg 2012; 77: 518-524.

7. Gourtsoyiannis N, Ros P. Radiologic-Pathologic Correlations from Head to Toe. Berlin/Heidelberg: Springer-Verlag; 2005.

8. Pérez-Ortiz L, Galarraga-Inza J, Gómez-Suárez H, TAmAYo-SuÁrez JD. Clasificación de los gliomas astrocíticos. Breves consideraciones. Rev Neurol 2000; 31: 1180-1183.

9. Vernooij MW, Ikram MA, Tanghe HL, Vincent AJPE, Hofman A, KRESTIN GP et al. Incidental findings on brain MRI in the general population. N Engl J Med 2007; 357: 1821-1828.

10. DeAngelis LM. Brain tumors. N Engl J Med 2001; 344: 114-123.

11. Ricard D, Idbaih A, Ducray F, Lahutte M, HoangXuan K, Delattre JY. Primary brain tumours in adults. Lancet 2012; 379: 1984-1996.

12. Fokas E, Steinbach JP, Rödel C. Biology of brain metastases and novel targeted therapies: Time to translate the research. Biochim Biophys Acta 2013; 1835: 61-75.

13. Albanese C, Rodriguez OC, Vanmeter J, Fricke ST, Rood BR, Lee Y et al. Preclinical magnetic resonance imaging and systems biology in cancer research: current applications and challenges. Am J Pathol 2013; 182: 312-318.

14. Erdem-Eraslan L, Gravendeel LA, De Rooi J, EILERS PHC, IDBAIH A, SPLIET WGM et al. Intrinsic molecular subtypes of glioma are prognostic and predict benefit from adjuvant procarbazine, lomustine, and vincristine chemotherapy in combination with other prognostic factors in anaplastic oligodendroglial brain tumors: a report from EORTC stu. J Clin Oncol 2013; 31: 328-336.
15. Helft PR. Am I obligated to give therapy against my better judgment? Oncology (Williston Park) 2012; 26: 995-996.

16. Kato Y, Nishihara H, Yuzawa S, Mohri H, Kanno $\mathrm{H}$, HATANAKA $\mathrm{Y}$ et al. Immunohistochemical molecular expression profile of metastatic brain tumor for potent personalized medicine. Brain Tumor Pathol 2012. Nov 20 (Epub ahead of print)

17. Lin SP, Lee YT, Wang JY, Miller SA, Chiou SH, Hung MC et al. Survival of cancer stem cells under hypoxia and serum depletion via decrease in PP2A activity and activation of p38MAPKAPK2-Hsp27. PloS One 2012; 7: e49605.

18. Neidert MC, Schoor O, Trautwein C, Trautwein N, Christ L, Melms A et al. Natural HLA class I ligands from glioblastoma: extending the options for immunotherapy. J Neurooncol 2013; 111: 285-294.

19. Bohan E, Glass-Macenka D. Surgical management of patients with primary brain tumors. Semin Oncol Nurs 2004; 20: 240-252.

20. BRicolo A. Surgical management of intrinsic brain stem gliomas. Oper Tech in Neurosurg 2000; 3: 137-154.

21. Demakas JJ, Sonntag VK, Kaplan AM, Kelley JJ, WAGGENER JD. Surgical management of pineal area tumors in early childhood. Surg Neurol 1982; 17: 435-440.

22. Drummond K, OJemann R. Surgical principles in the management of brain tumors. Brain Tumors, Edinburgh: W.B. Saunders; 2012; 282295.

23. Fasano VA, Urciudol R, Ponzio RM, Lanotte MM. The effects of new technologies on the surgical management of brainstem tumors. Surg Neurol 1986; 25: 219-226.

24. Frazier J, Jallo G. Chapter 13 - Surgical Management of Brain Stem Tumors in Adults. Schmidek and Sweet's Operative Neurosurgical Techniques, Philadelphia: W.B. Saunders; 2012; 160-168.

25. Jallo G, Kothbauer K, Epstein F. Surgical management of cervicomedullary and dorsally exophytic brain stem tumors. Oper Techniq Neurosurg 2000; 3: 131-136.

26. Kellner CP, D’Ambrosio AL. Surgical management of brain metastases. Neurosurg Clin N Am 2011; 22: 53-59, vi.

27. Lang SS, Beslow LA, Gabel B, Judkins AR, Fisher MJ, SuTTON LN et al. Surgical treatment of brain tumors in infants younger than six months of age and review of the literature. World Neurosurg 2012; 78: 137-144. 
28. Pant B, Shrestha P. Management of brain tumor in Nepal. Int Congr Ser 2004; 1259: 149155.

29. Patel TR, Yu JB, Piepmeier JM. Role of neurosurgery and radiation therapy in the management of brain tumors. Hematol Oncol Clin North Am 2012; 26: 757-777.

30. Wen PY, Schiff D, Kesari S, Drappatz J, Gigas DC, DoHERTY L. Medical management of patients with brain tumors. J Neurooncol 2006; 80: 313-332.

31. Ferson D, DeMonte F. Anesthetic management of patients undergoing surgery for brain tumors. Anesthesiology Clin N Am 1998; 16: 663-675.

32. KaAKeh Y, Overholser BR, Lopshire JC, Tisdale JE. Drug-induced atrial fibrillation. Drugs 2012; 72: 1617-1630.

33. Kharin SN, Krandycheva VV, Strelkova MV, TsVETKOVA AS, ShmaKov DN. Doxorubicin-induced changes of ventricular repolarization heterogeneity: results of a chronic rat study. Cardiovasc Toxicol 2012; 12: 312-317.

34. Ratterree W, Gieger T, Pariaut R, Saelinger C, STRICKLAND K. Value of echocardiography and electrocardiography as screening tools prior to Doxorubicin administration. J Am Anim Hosp Assoc 2012; 48: 89-96.

35. Xin Y, Zhang S, Gu L, Liu S, GaO H, You Z et al. Electrocardiographic and biochemical evidence for the cardioprotective effect of antioxidants in acute doxorubicin-induced cardiotoxicity in the beagle dogs. Biol Pharm Bull 2011; 34: 1523-1526.

36. Paun A, Lemay AM, Tomko TG, Haston CK. Association analysis reveals genetic variation altering bleomycin-induced pulmonary fibrosis in mice. Am J Respir Cell Mol Biol 2013; 48: 330-336.

37. Trivedi R, Redente EF, Thakur A, Riches DWH, Kompella UB. Local delivery of biodegradable pirfenidone nanoparticles ameliorates bleomycin-induced pulmonary fibrosis in mice. Nanotechnology 2012; 23: 505101.

38. Burns WA, McFarland W, Matthews MJ. Busulfan-induced pulmonary disease. Report of a case and review of the literature. Am Rev Respir Dis 1970; 101: 408-413.

39. Guy C, Vergnon JM, Vasselon C, Ollagnier M. Fibrose pulmonaire au busulfan. Thérapie 1986; 41: 315-316.

40. Massin F, Fur A, Reybet-Degat O, Camus P, JeanNIN L. [Busulfan-induced pneumopathy]. Rev Mal Respir 1987; 4: 3-10.
41. Chen H-H, Chen T-W, Lin H. Pravastatin attenuates carboplatin-induced nephrotoxicity in rodents via peroxisome proliferator-activated receptor alpha-regulated heme oxygenase-1. Mol Pharmacol 2010; 78: 36-45.

42. Donadio C, Lucchesi A, Ardini M, Cosio S, FanucCHI A, GADDUCCI A. Dose individualization can minimize nephrotoxicity due to carboplatin therapy in patients with ovarian cancer. Ther Drug Monit 2009; 31: 63-69.

43. Fujieda M, Matsunaga A, Hayashi A, Tauchi $\mathrm{H}$, Chayama K, Sekine T. Children's toxicology from bench to bed-Drug-induced renal injury (2): Nephrotoxicity induced by cisplatin and ifosfamide in children. J Toxicol Sci 2009; 34 Suppl 2: SP 251-257.

44. Kintzel PE. Anticancer drug-induced kidney disorders. Drug Saf 2001; 24: 19-38.

45. Korkmaz A, Topal T, Oter S. Pathophysiological aspects of cyclophosphamide and ifosfamide induced hemorrhagic cystitis; implication of reactive oxygen and nitrogen species as well as PARP activation. Cell Biol Toxicol 2007; 23: 303-312.

46. Macedo FYB, Mourão LTC, Freitas HC, Lima RCP, WONG DVT, ORIÁ RB et al. Interleukin-4 modulates the inflammatory response in ifosfamide-induced hemorrhagic cystitis. Inflammation 2012;35:297-307.

47. Mota JM, Brito GA, Loiola RT, Cunha FQ, RibeiRO R DE A. Interleukin-11 attenuates ifosfamide-induced hemorrhagic cystitis. Int Braz J Urol 2007; 33: 704-710.

48. Vieira MM, Brito GAC, Belarmino-Filho JN, MACEDO FYBM, NERY EA, CunHA FQ et al. Use of dexamethasone with mesna for the prevention of ifosfamide-induced hemorrhagic cystitis. Int J Urol 2003; 10: 595-602.

49. Al-Jafari AA, Duhaiman AS, Kamal MA. Inhibition of human acetylcholinesterase by cyclophosphamide. Toxicology 1995; 96: 1-6.

50. Al-Jafari AA, Shakil S, Reale M, Kamal MA. Human platelet acetylcholinesterase inhibition by cyclophosphamide: a combined experimental and computational approach. CNS Neurol Disord Drug Targets 2011; 10: 928-935.

51. Bodur E. Human serum butyrylcholinesterase interactions with cisplatin and cyclophosphamide. Biochimie 2010; 92: 979-984.

52. Kamal MA, Reale M, Al-Jafari AA. Multiple approaches to analyse the data for rat brain acetylcholinesterase inhibition by cyclophosphamide. Neurochem Res 2010; 35: 1501-1509. 
53. Shakil S, Kamal MA, Tabrez S, Abuzenadah AM, Chaudhary AGA, Damanhouri GA. Molecular interaction of the antineoplastic drug, methotrexate with human brain acetylcholinesterase: a docking study. CNS Neurol Disord Drug Targets 2012; 11: 142-147.

54. Pilgrim CHC, Thomson BN, Banting S, Phillips WA, Michael M. The developing clinical problem of chemotherapy-induced hepatic injury. ANZ Journal of Surgery 2012; 82: 23-29.

55. Carr C, NG J, Wigmore T. The side effects of chemotherapeutic agents. Curr Anaesth Crit Care 2008; 19: 70-79.

56. Floyd J, Mirza I, Sachs B, Perry MC. Hepatotoxicity of chemotherapy. Semin Oncol 2006; 33 : 50-67.

57. Fleisher LA, Beckman JA, Brown KA, Calkins $\mathrm{H}$, Chaikof EL, Chaikof E et al. ACC/AHA 2007 guidelines on perioperative cardiovascular evaluation and care for noncardiac surgery: a report of the American College of Cardiology/American Heart Association Task Force on Practice Guidelines (Writing Committee to Revise the 2002 Guideline. J Am Coll Cardiol 2007; 50: e159-241.

58. Fleisher la, Beckman JA, Brown KA, Calkins H, Chaikof EL, Fleischmann KE et al. 2009 ACCF/ AHA focused update on perioperative beta blockade incorporated into the ACC/AHA 2007 guidelines on perioperative cardiovascular evaluation and care for noncardiac surgery. J Am Coll Cardiol 2009; 54: e13-e118.

59. SAlEs Llopis J. Evaluación preoperatoria en Neurocirugía, Nociones de neuroanestesia, Evaluación neurofisiológica preoperatoria. Rev Neurocirugía Contemporánea 2009; 3; 186-197.

60. Cameron MH, Klein EL. Screening for medical disease-nervous system disorders. J Hand Ther 2010; 23: 158-171.

61. Terret C, Albrand G, Moncenix G, Droz JP. KarNOFSKY Performance Scale (KPS) or Physical Performance Test (PPT)? That is the question. Crit Rev Oncol Hematol 2011; 77: 142147.

62. Ianssen T, Lydersen S, Torp S, R J. Malignant brain tumors-effect of repeated resection and adjuvant treatment. Int Congr Ser 2004; 1259: 45-52.

63. Karnofsky D, Burchenal J. The Clinical Evaluation of Chemotherapeutic Agents in Cancer. Columbia Univ Press; 1949.

64. Dowling C, Bollen AW, Noworolski SM, McDermott MW, Barbaro NM, Day MR et al. Preoperative proton $\mathrm{MR}$ spectroscopic imaging of brain tumors: correlation with histopathologic analysis of resection specimens. Am J Neuroradiol 2001; 22: 604-612.

65. Kamada K, Todo T, Masutani Y, Aoki S, Ino K, TAKANO $\mathrm{T}$ et al. Combined use of tractography-integrated functional neuronavigation and direct fiber stimulation. J Neurosurg 2005; 102: 664-672.

66. Kamada K, Todo T, Masutani Y, Aoki S, Ino K, Morita A et al. Visualization of the frontotemporal language fibers by tractography combined with functional magnetic resonance imaging and magnetoencephalography. J Neurosurg 2007; 106: 90-98.

67. Arbizu J, Domínguez PD, Diez-Valle R, Vigil C, García-Eulate R, Zubieta JL et al. Neuroimagen en tumores cerebrales. Rev Esp Med Nucl 2011; 30: 47-65.

68. Bagadia A, Purandare H, Misra BK, Gupta S. Application of magnetic resonance tractography in the perioperative planning of patients with eloquent region intra-axial brain lesions. J Clin Neurosci 2011; 18: 633-639.

69. Berntsen EM, Gulati S, Solheim O, Kvistad KA, Torp SH, SelbekK T et al. Functional magnetic resonance imaging and diffusion tensor tractography incorporated into an intraoperative 3-dimensional ultrasound-based neuronavigation system: impact on therapeutic strategies, extent of resection, and clinical outcome. Neurosurgery 2010; 67: 251-264.

70. Kuhnt D, Bauer MHA, Becker A, Merhof D, ZoLAL A, Richter M et al. Intraoperative visualization of fiber tracking based reconstruction of language pathways in glioma surgery. Neurosurgery 2012; 70: 911-919; discussion 919920.

71. Majchrzak K, Bobek-Billewicz B, Tymowski M, Adamczyk P, Majchrzak H, Ladzi Ski P. Surgical treatment of insular tumours with tractography, functional magnetic resonance imaging, transcranial electrical stimulation and direct subcortical stimulation support. Neurol Neurochir Pol 2011; 45: 351-362.

72. Prabhu SS, Gasco J, Tummala S, Weinberg JS, RAO G. Intraoperative magnetic resonance imaging-guided tractography with integrated monopolar subcortical functional mapping for resection of brain tumors. Clinical article. J Neurosurg 2011; 114: 719-726.

73. Zhao Y, Chen X, Wang F, Sun G, Wang Y, Song $Z$ et al. Integration of diffusion tensor-based arcuate fasciculus fibre navigation and intraoperative MRI into glioma surgery. J Clin Neurosci 2012; 19: 255-261. 
74. Zhao Y, Chen X-L, Wang F, Sun G-C, Wang Y-B, SONG Z-J et al. [Application of diffusion tensor imaging-based arcuate fasciculus tractography and intraoperative arcuate fasciculus navigation]. Zhongguo Yi Xue Ke Xue Yuan Xue Bao 2011; 33: 499-503.

75. Krieg SM, Ringel F, Meyer B. Functional guidance in intracranial tumor surgery. Per Med 2012; 1: 59-64.

76. Abe T, Black PM, Ojemann RG, Hedley-White ET. Cerebral edema in intracranial meningiomas: evidence for local and diffuse patterns and factors associated with its occurrence. Surg Neurol 1994; 42: 471-475.

77. HASso AN, Bell SA, TADMOR R. Intracranial vascular tumors. Neuroimaging Clin N Am 1994; 4: $849-870$.

78. Papadopoulos MC, Saadoun S, Binder DK, Manley GT, KRISHna S, Verkman AS. Molecular mechanisms of brain tumor edema. Neuroscience 2004; 129: 1011-1020.

79. Hardwidge C, Hettige S. Tumours of the central nervous system. Surgery (Oxford) 2012; 30: 7.

80. Van Breemen MSM, Wilms EB, Vecht CJ. Epilepsy in patients with brain tumours: epidemiology, mechanisms, and management. Lancet Neurol 2007; 6: 421-430.

81. Lote K, Stenwig AE, Skullerud K, Hirschberg H. Prevalence and prognostic significance of epilepsy in patients with gliomas. Eur J Cancer 1998; 34: 98-102.

82. Vecht CJ, Wagner GL, WiLms EB. Treating seizures in patients with brain tumors: Drug interactions between antiepileptic and chemotherapeutic agents. Semin Oncol 2003; 30: 49-52.

83. Vecht CJ, Van Breemen M. Optimizing therapy of seizures in patients with brain tumors. Neurology 2006; 67: S10-13.

84. Boarini DJ, Beck DW, VAnGILder JC. Postoperative prophylactic anticonvulsant therapy in cerebral gliomas. Neurosurgery 1985;16:2902.

85. Cohen N, Strauss G, Lew R, Silver D, Recht L. Should prophylactic anticonvulsants be administered to patients with newly-diagnosed cerebral metastases? A retrospective analysis. J Clin Oncol 1988; 6: 1621-1624.

86. Mahaley MS, DudKa L. The role of anticonvulsant medications in the management of patients with anaplastic gliomas. Surg Neurol 1981; 16: 399-401.

87. De Santis A, Villani R, Sinisi M, Stocchetti N, Perucca E. Add-on phenytoin fails to prevent early seizures after surgery for supratentorial brain tumors: a randomized controlled study. Epilepsia 2002; 43: 175-182.

88. Patsalos PN, Fröscher W, Pisani F, Van Rijn CM. The importance of drug interactions in epilepsy therapy. Epilepsia 2002; 43: 365-385.

89. Fleming AJ, ChI SN. Brain tumors in children. Curr Probl Pediatr Adolesc Health Care 2012; 42: 80-103.

90. Lynam LM, Lyons MK, DRAZKowSKi JF, SiRVEN JI, NoE KH, Zimmerman RS et al. Frequency of seizures in patients with newly diagnosed brain tumors: a retrospective review. Clin Neurol Neurosurg 2007; 109: 634-638.

91. Wolf HK, Roos D, Blümcke I, Pietsch T, Wiestler OD. Perilesional neurochemical changes in focal epilepsies. Acta Neuropathol 1996; 91: 376-384.

92. Kahlenberg CA, Fadul CE, Roberts DW, Thadani VM, Bujarski KA, ScotT RC et al. Seizure prognosis of patients with low-grade tumors. Seizure 2012;21:540-5.

93. Tremont-Lukats IW, Ratilal BO, Armstrong T, GILBERT MR. Antiepileptic drugs for preventing seizures in people with brain tumors. Cochrane Database Syst Rev 2008: CD004424.

94. Kerrigan S, Grant R. Antiepileptic drugs for treating seizures in adults with brain tumours. Cochrane Database Syst Rev 2011: CD008586.

95. Lim DA, Tarapore P, Chang E, Burt M, ChaKaLIAN L, BARbaro $\mathrm{N}$ et al. Safety and feasibility of switching from phenytoin to levetiracetam monotherapy for glioma-related seizure control following craniotomy: a randomized phase II pilot study. J Neurooncol 2009; 93 : 349-354.

96. Wu AS, Trinh VT, Suki D, Graham S, Forman A, WeINBERG JS et al. A prospective randomized trial of perioperative seizure prophylaxis in patients with intraparenchymal brain tumors. J Neurosurg 2013.

97. Komotar RJ, Raper DMS, Starke RM, Iorgulescu JB, Gutin PH. Prophylactic antiepileptic drug therapy in patients undergoing supratentorial meningioma resection: a systematic analysis of efficacy. J Neurosurg 2011; 115: 483-490.

98. Hardesty DA, SAnborn MR, Parker WE, Storm PB. Perioperative seizure incidence and risk factors in 223 pediatric brain tumor patients without prior seizures. J Neurosurg Pediatr 2011; 7: 609-615.

99. Trousseau A. Phlegmasia alba dolens. Clin Med Hotel Dieu De Paris 1865; 3: 94-96. 
100. Heit JA, Silverstein MD, Mohr DN, Petterson TM, O'FALLON WM, MELTON LJ. Risk factors for deep vein thrombosis and pulmonary embolism: a population-based case-control study. Arch Intern Med 2000; 160: 809-815.

101. Silverstein MD, Heit JA, Mohr DN, Petterson TM, O'Fallon WM, Melton LJ. Trends in the incidence of deep vein thrombosis and pulmonary embolism: a 25-year populationbased study. Arch Intern Med 1998; 158: 585-593.

102. Preusser M, De Ribaupierre S, Wöhrer A, ErridGe SC, Hegi M, Weller M et al. Current concepts and management of glioblastoma. Ann Neurol 2011; 70: 9-21.

103. Brandes AA, Scelzi E, Salmistraro G, Ermani M, CARollo C, Berti $\mathrm{F}$ et al. Incidence of risk of thromboembolism during treatment highgrade gliomas: a prospective study. Eur J Cancer 1997; 33: 1592-1596.

104. SJöBlom L, HÅrdemark HG, Lindgren A, NorRVING B, FAHLÉN M, SAMUELSSON M et al. Management and prognostic features of intracerebral hemorrhage during anticoagulant therapy: a Swedish multicenter study. Stroke 2001; 32: 2567-2574.

105. Hylek EM, Singer DE. Risk factors for intracranial hemorrhage in outpatients taking warfarin. Ann Intern Med 1994; 120: 897-902.

106. Perry JR. Anticoagulation of malignant glioma patients in the era of novel antiangiogenic agents. Curr Opin Neurol 2010; 23: 592596.

107. LEE AYY. Epidemiology and management of venous thromboembolism in patients with cancer. Thrombosis Research 2003; 110: 167-172.

108. Horsted F, West J, Grainge MJ. Risk of venous thromboembolism in patients with cancer: a systematic review and meta-analysis. PLoS Med 2012; 9: e1001275.

109. Collen JF, Jackson JL, Shorr AF, Moores LK. Prevention of venous thromboembolism in neurosurgery: a metaanalysis. Chest 2008; 134: 237-249.

110. Geerts WH, Bergqvist D, Pineo GF, Heit JA, Samama CM, Lassen MR et al. Prevention of venous thromboembolism: American College of Chest Physicians Evidence-Based Clinical Practice Guidelines (8th Edition). Chest 2008; 133: 381S-453S.

111. Muntz JE, Michota FA. Prevention and management of venous thromboembolism in the surgical patient: options by surgery type and individual patient risk factors. Am J Surg 2010; 199: S11-20.
112. Ten Cate-Hoek AJ, Prins MH. Low molecular weight heparins in cancer: Management and prevention of venous thromboembolism in patients with malignancies. Thromb Res 2008; 122: 584-598.

113. Imberti D, Di Nisio M, Donati MB, Falanga A, GhirarduZZI A, GuARnERI D et al. Treatment of venous thromboembolism in patients with cancer: Guidelines of the Italian Society for Haemostasis and Thrombosis (SISET). Thromb Res 2009; 124: e32-e40.

114. Khemasuwan D, DiVietro ML, TangdhanaKanond K, Pomerantz SC, Eiger G. Statins decrease the occurrence of venous thromboembolism in patients with cancer. Am J Med 2010; 123: 60-65.

115. Lyman GH, Khorana AA, Falanga A, ClarkePearson D, Flowers C, Jahanzeb M et al. American Society of Clinical Oncology guideline: recommendations for venous thromboembolism prophylaxis and treatment in patients with cancer. J Clin Oncol 2007; 25: 5490-5505.

116. LuKINS MB, MANNINEN PH. Hyperglycemia in patients administered dexamethasone for craniotomy. Anesth Analg 2005; 100: 11291133.

117. Hans P, Vanthuyne A, Dewandre Py, Brichant JF, Bonhomme V. Blood glucose concentration profile after $10 \mathrm{mg}$ dexamethasone in non-diabetic and type 2 diabetic patients undergoing abdominal surgery. $\mathrm{Br} \mathrm{J}$ Anaesth 2006; 97: 164-170.

118. McGirt MJ, Chaichana KL, Gathinji M, AtteneLlo F, Than K, Ruiz AJ et al. Persistent outpatient hyperglycemia is independently associated with decreased survival after primary resection of malignant brain astrocytomas. Neurosurgery 2008; 63: 286-291; discussion 291.

119. Derr RL, Ye X, Islas MU, Desideri S, Saudek CD, Grossman SA. Association between hyperglycemia and survival in patients with newly diagnosed glioblastoma. J Clin Oncol 2009; 27: 1082-1086.

120. Seyfried TN, SAnderson TM, El-Abbadi MM, McGowan R, Mukherjee P. Role of glucose and ketone bodies in the metabolic control of experimental brain cancer. Br J Cancer 2003; 89: 1375-1382.

121. Seyfried TN, Mukherjee P. Targeting energy metabolism in brain cancer: review and hypothesis. Nutr Metab (Lond) 2005; 2: 30.

122. Peca C, Pacelli R, Elefante A, Del Basso De Caro ML, Vergara P, Mariniello G et al. Early clinical and neuroradiological worsening after radiotherapy and concomitant temo- 
zolomide in patients with glioblastoma: tumour progression or radionecrosis? Clin Neurol Neurosurg 2009; 111: 331-334.

123. Di Chiro G, Oldfield E, Wright DC, De Michele D, Katz DA, Patronas NJ et al. Cerebral necrosis after radiotherapy and/or intraarterial chemotherapy for brain tumors: PET and neuropathologic studies. AJR Am J Roentgenol 1988; 150: 189-197.

124. Lee WH, Sonntag We, Mitschelen M, Yan $\mathrm{H}$, LEE YW. Irradiation induces regionally specific alterations in pro-inflammatory environments in rat brain. Int $\mathrm{J}$ Radiat Biol 2010; 86: 132-144.

125. Takano T, Lin JH, Arcuino G, Gao Q, Yang J, NEDERGAARD M. Glutamate release promotes growth of malignant gliomas. Nat Med 2001; 7: 1010-1015.

126. Hawkins RA, Mokashi A, Dejoseph MR, Viña JR, FERNSTROM JD. Glutamate permeability at the blood-brain barrier in insulinopenic and insulin-resistant rats. Metabolism 2010; 59: 258-266.

127. Yang C, Sudderth J, Dang T, Bachoo RM, Bachoo RG, McDonald JG et al. Glioblastoma cells require glutamate dehydrogenase to survive impairments of glucose metabolism or Akt signaling. Cancer Res 2009; 69: 7986-7993.

128. DeBerardinis RJ, Mancuso A, DaiKhin E, Nissim I, Yudkoff M, Wehrl $S$ et al. Beyond aerobic glycolysis: transformed cells can engage in glutamine metabolism that exceeds the requirement for protein and nucleotide synthesis. Proc Natl Acad Sci U S A 2007; 104: 19345-19350.

129. Newsholme P. Why is L-glutamine metabolism important to cells of the immune system in health, postinjury, surgery or infection? J Nutr 2001; 131: 2515S-2522S; discussion 2523S-2524S.

130. DeBerardinis RJ, Cheng T. Q's next: the diverse functions of glutamine in metabolism, cell biology and cancer. Oncogene 2010; 29: 313-324.

131. Greene AE, Todorova MT, Seyfried TN. Perspectives on the metabolic management of epilepsy through dietary reduction of glucose and elevation of ketone bodies. J Neurochem 2003; 86: 529-537.

132. WEINDRUCH R. The retardation of aging by caloric restriction: studies in rodents and primates. Toxicol Pathol 1996; 24: 742-745.

133. BiRT DF, Yaktine A, DuYsen E. Glucocorticoid mediation of dietary energy restriction inhibition of mouse skin carcinogenesis. J Nutr 1999; 129: 571S-574S.

134. Duan W, Lee J, Guo Z, Mattson MP. Dietary restriction stimulates BDNF production in the brain and thereby protects neurons against excitotoxic injury. $\mathrm{J}$ Mol Neurosci 2001; 16: 1-12. 